\title{
SXP 1062, a young Be X-ray binary pulsar with long spin period ${ }^{\star}$ Implications for the neutron star birth spin
}

\author{
F. Haberl ${ }^{1}$, R. Sturm ${ }^{1}$, M. D. Filipovic ${ }^{2}$, W. Pietsch ${ }^{1}$, and E. J. Crawford ${ }^{2}$ \\ 1 Max-Planck-Institut für extraterrestrische Physik, Giessenbachstraße, 85748 Garching, Germany \\ e-mail: fwh@mpe.mpg.de \\ 2 University of Western Sydney, Locked Bag 1797, Penrith South DC, NSW1797, Australia
}

Received 31 October 2011 / Accepted 1 December 2011

\section{ABSTRACT}

\begin{abstract}
Context. The Small Magellanic Cloud (SMC) is ideally suited to investigating the recent star formation history from X-ray source population studies. It harbours a large number of Be/X-ray binaries (Be stars with an accreting neutron star as companion), and the supernova remnants can be easily resolved with imaging X-ray instruments.

Aims. We search for new supernova remnants in the SMC and in particular for composite remnants with a central X-ray source.

Methods. We study the morphology of newly found candidate supernova remnants using radio, optical and X-ray images and investigate their X-ray spectra.

Results. Here we report on the discovery of the new supernova remnant around the recently discovered Be/X-ray binary pulsar CXO J012745.97-733256.5 = SXP 1062 in radio and X-ray images. The Be/X-ray binary system is found near the centre of the supernova remnant, which is located at the outer edge of the eastern wing of the SMC. The remnant is oxygen-rich, indicating that it developed from a type Ib event. From XMM-Newton observations we find that the neutron star with a spin period of $1062 \mathrm{~s}$ (the second longest known in the SMC) shows a very high average spin-down rate of $0.26 \mathrm{~s}$ per day over the observing period of 18 days. Conclusions. From the currently accepted models, our estimated age of around 10 000-25000 years for the supernova remnant is not long enough to spin down the neutron star from a few $10 \mathrm{~ms}$ to its current value. Assuming an upper limit of 25000 years for the age of the neutron star and the extreme case that the neutron star was spun down by the accretion torque that we have measured during the XMM-Newton observations since its birth, a lower limit of $0.5 \mathrm{~s}$ for the birth spin period is inferred. For more realistic, smaller long-term average accretion torques our results suggest that the neutron star was born with a correspondingly longer spin period. This implies that neutron stars in Be/X-ray binaries with long spin periods can be much younger than currently anticipated.
\end{abstract}

Key words. stars: neutron - X-rays: binaries - Magellanic Clouds - stars: emission-line, Be

\section{Introduction}

One of the most cataclysmic events in the universe is the explosion of a massive star as a supernova, which can create a neutron star (NS). NSs are thought to be born rapidly spinning with rotation periods of a few $10 \mathrm{~ms}$. Their rotation is first slowed down by magnetic dipole braking and then by the propeller effect when the NS is in a binary star system and mass loss from the companion star begins (for a review see e.g. Bhattacharya \& van den Heuvel 1991). When rotating slowly enough, accretion onto the NS sets in (Ghosh \& Lamb 1978) and the system can be detected as an X-ray binary. The spin evolution of the NS in a high-mass X-ray binary (HMXB) system depends on the initial magnetic field strength of the NS and on the mass accretion rate (Urpin et al. 1998).

Be/X-ray binary (BeXRB) systems are a subgroup of HMXBs with an NS accreting matter from the circumstellar disk of a Be star (Reig 2011). The Small Magellanic Cloud (SMC) harbours an extraordinarily large number of BeXRBs (Coe et al. 2005; Shtykovskiy \& Gilfanov 2005; Haberl et al. 2008). These are believed to have been created during a burst of star

* Based on observations with XMM-Newton, an ESA Science Mission with instruments and contributions directly funded by ESA Member states and the USA (NASA). formation about 42 million years ago (Antoniou et al. 2010). For more than fifty BeXRBs in the SMC the NS spin period is known from the detection of coherent pulsations in their X-ray flux. The spin periods range from 2.16 s (XTE J0119-731 = SXP 2.16; Corbet et al. 2003) to $1320 \mathrm{~s}$ (RX J0103.6-7201 = SXP 1323; Haberl \& Pietsch 2005) with a bimodal structure indicated in their distribution (Knigge et al. 2011).

From ROSAT HRI data, Hughes \& Smith (1994) proposed two BeXRBs within supernova remnants (SNRs) in the SMC. For one of them, located in the direction of the SNR IKT 21 (van der Heyden et al. 2004), pulsations of 345 s were discovered by Israel et al. (2000, SAX J0103.2-7209). The other - still unconfirmed - candidate BeXRB in the direction of IKT 25 was not detected by XMM-Newton or Chandra. In both cases it is not clear whether the BeXRB (or candidate) is physically associated with the SNR because they are not located near the centre of the remnant and the high number of BeXRBs in the bar of the SMC make chance coincidences likely. The SNR, which was created by the supernova explosion, is expected to have faded beyond detectability before accretion onto the NS starts. Finding a BeXRB associated to a SNR would therefore suggest a much faster spin-down than predicted by the standard models, or else the NS star was born with slow rotation. Here, we present the discovery of an SNR in radio and X-rays around the BeXRB 

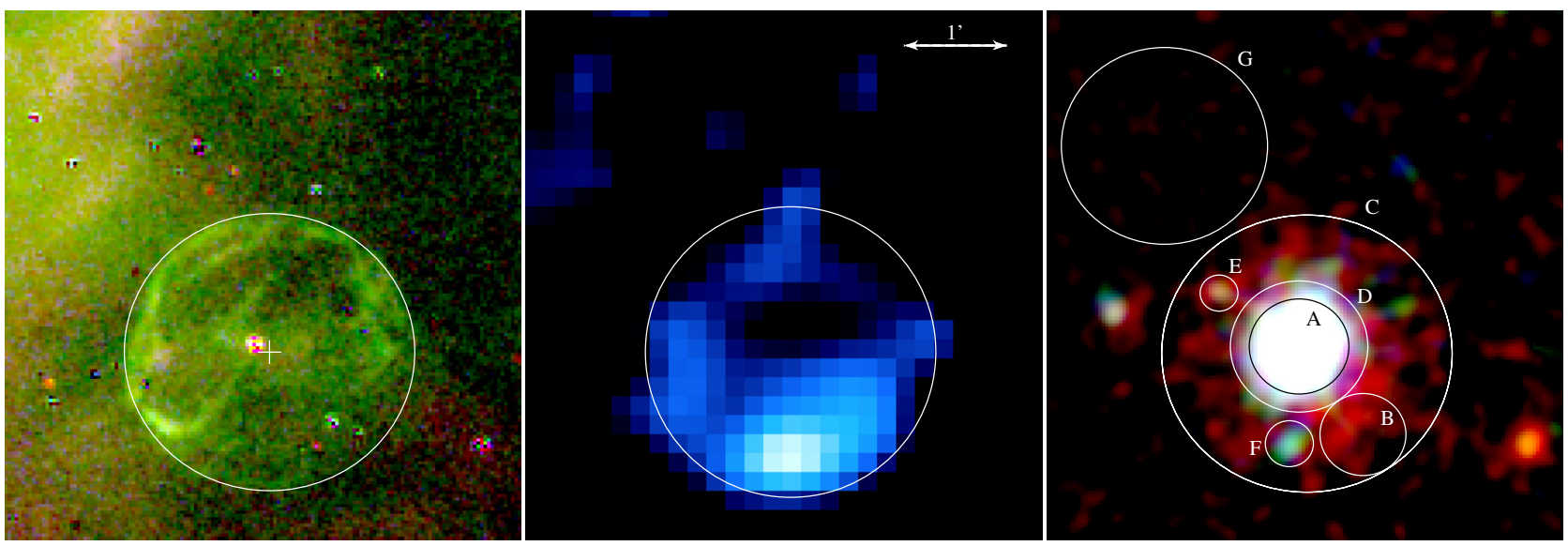

Fig. 1. Images of the region around the BeXRB SXP 1062. Left: continuum-subtracted MCELS images. Red, green, and blue correspond to H $\alpha$, $\left[\mathrm{O}_{\mathrm{III}}\right]$ and $\left[\mathrm{S}_{\mathrm{II}}\right]$. The cross marks the centre of the white circle $\left(\mathrm{RA}=01^{\mathrm{h}} 27^{\mathrm{m}} 44^{\mathrm{s}} .15\right.$, Dec $=-73^{\circ} 33^{\prime} 01^{\prime \prime} 6$, J2000), which indicates the estimated size of the SNR in all images. The Be star counterpart of SXP 1062 is visible close to the cross. Middle: MOST $36 \mathrm{~cm}$ radio image, logarithmically scaled from 0.5 to $3 \mathrm{mJy}$. Right: combined, instrument background-subtracted EPIC-pn and MOS colour image obtained from the XMM-Newton observations. Red, green, and blue denote X-ray intensities in the $0.2-1.0,1.0-2.0$, and $2.0-4.5 \mathrm{keV}$ bands. The images from the individual bands were adaptively smoothed with a Gaussian $\sigma$ of $4^{\prime \prime}$ (at high intensities) to 20" (low intensities). Circles indicate the extraction regions for the X-ray spectra: Source region for SXP 1062 (A, 29" radius) and associated background (B, 25"). For the SNR spectrum (C, 84.5"), regions D (40"), E $\left(11^{\prime \prime}\right)$, and $\mathrm{F}\left(14^{\prime \prime}\right)$ were excluded and region $\mathrm{G}\left(60^{\prime \prime}\right)$ was used to estimate the background. Regions $\mathrm{E}$ and $\mathrm{F}$ were excluded because they contain possible point sources in the direction of the SNR as found in source detection runs on the EPIC images.

pulsar CXO J012745.97-733256.5 = SXP 1062. The discovery of the new SMC pulsar and its possible association with a shell nebula around it has recently been reported by Henault-Brunet et al. (2011, hereafter HB11).

\section{Multi-wavelength observations and data analysis}

\subsection{Optical}

The Magellanic Cloud Emission Line Survey ${ }^{1}$ was carried out from the $0.6 \mathrm{~m}$ University of Michigan/CTIO Curtis Schmidt telescope, equipped with an SITE $2048 \times 2048$ CCD. This resulted in a field of $1.35^{\circ} \times 1.35^{\circ}$ with a pixel scale of $2.4^{\prime \prime} \times$ $2.4^{\prime \prime}$. The Magellanic Clouds were mapped in narrow wavelength bands corresponding to $\mathrm{H} \alpha$, [O $\mathrm{OII}]$, and [ $\left.\mathrm{S}_{\mathrm{II}}\right]$, together with matching red and green continuum bands. All the images were continuum-subtracted, flux-calibrated, and assembled into mosaic images. A composite image in the three bands of the region around SXP 1062 is presented in Fig. 1, revealing a shelltype nebula dominated by the strong [O III] line as also discussed by HB11.

\subsection{Radio-continuum}

The field of SXP 1062 was observed with the Australia Telescope Compact Array (ATCA) on 2009-01-05 with array configuration 6C, and on 2009-02-05 in array configuration EW352 (ATCA project C1869). The field was observed at wavelengths of $20 \mathrm{~cm}$ and $13 \mathrm{~cm}$. Each session was carried out in "snap-shot" mode, totalling $\sim 3.5 \mathrm{~h}$ of integration time over a $12 \mathrm{~h}$ period. These data were combined with the relevant parts of the data from Wong et al. (2011) and Filipovic et al. (1995) to improve $u-v$ plane coverage. The $13 \mathrm{~cm}$ data proved unusable due to radio frequency interference. The $20 \mathrm{~cm}$ data was useful only when long baselines were excluded thus the final image has a resolution of $145^{\prime \prime} \times 131^{\prime \prime}$ with an r.m.s. noise of $0.5 \mathrm{mJy} / \mathrm{beam}$. We report an integrated flux density of $6 \mathrm{mJy}$ at $20 \mathrm{~cm}$.

1 MCELS: http://www.ctio.noao.edu/ mcels/
Using the image of Ye \& Turtle (1993), we measured an integrated flux density of $9 \mathrm{mJy}$ at $36 \mathrm{~cm}$ (see Fig. 1). Combining this measurement with the $20 \mathrm{~cm}$ measurement we derive a spectral index $\left(S_{v} \propto v^{\alpha}\right)$ of $\alpha=-0.8 \pm 0.4$ which is steeper than most SNRs in the Magellanic Clouds (Filipovic et al. 1998). We estimate that the surface brightness of this SNR is $1.6 \times$ $10^{-22} \mathrm{~W} \mathrm{~m}^{-2} \mathrm{~Hz}^{-1} \mathrm{Sr}^{-1}$ with a luminosity of $3.4 \times 10^{15} \mathrm{~W} \mathrm{~Hz}^{-1}$, assuming a distance of $60 \mathrm{kpc}$ and diameter of $166^{\prime \prime}$. This makes it the faintest known radio SNR in the SMC (Filipović et al. 2005, 2008; Owen et al. 2011).

\subsection{X-rays}

Chandra (between 2010-03-31 and 2010-04-29) and XMM-Newton (2010-03-25 to 2010-04-12) observed the field around the star-forming region NGC 602 and revealed the new BeXRB SXP 1062 in the eastern wing of the SMC (HB11) with a spin period of $1062 \mathrm{~s}$, the second longest known in the SMC after SXP 1323. Here, we present the analysis of the four XMM-Newton observations (observation IDs 0602520401, 0602520201, 0602520301, and 0602520501) with durations of $66.3,119.0,91.2$, and $55.0 \mathrm{ks}$ and spread over 18 days.

\subsubsection{Morphology}

Following the analysis of the SMC survey with XMM-Newton (Haberl et al., in prep.; Sturm et al., in prep.), we produced X-ray images from the combined EPIC-pn (Strüder et al. 2001) and EPIC-MOS (Turner et al. 2001) data in the energy bands $0.2-1.0 \mathrm{keV}, 1.0-2.0 \mathrm{keV}$, and $2.0-4.5 \mathrm{keV}$. Our analysis of the EPIC data used the tools from the XMM-Newton SAS package version $11.0 .1^{2}$. To optimise the sensitivity for faint diffuse X-ray emission we removed intervals of high background caused by soft protons and subtracted the contribution of the detector particle background from the images. For EPIC-pn also

\footnotetext{
${ }^{2}$ Science Analysis Software (SAS), http://xmm.esac.esa.int/ sas/
} 
the out-of-time events recorded during CCD readout were subtracted. The final colour image of the region around SXP 1062 is presented in Fig. 1. Although the emission from SXP 1062 dominates the hard energy bands, soft extended emission is clearly seen around the X-ray pulsar. The extent of this emission is similar to the radio and MCELS images. We also checked the Chandra images for the soft emission, but the sensitivity at low energies is insufficient for the Chandra ACIS-I instrument to allow a significant detection.

\subsubsection{Spectral analysis}

We extracted EPIC spectra from the X-ray pulsar and the soft emission region, merging the data from all four observations. The extraction regions are indicated in Fig. 1 and described below. No MOS1 data is available because the source was located on CCD 6, which is no longer operative. For the spectra we selected single and double-pixel events (PATTERN 0-4) for pn and single to quadruple events (PATTERN 0-12) for MOS with FLAG $=0$ to avoid bad pixels. Applying the same background flare screening as for the images resulted in net exposures for the spectra of $206.8 \mathrm{ks}$ for pn and $236.7 \mathrm{ks}$ for MOS2.

To extract the spectra of SXP 1062 we used a circular region and a very close background region inside the soft emission region to remove the contribution of this component. We fitted the pn and MOS2 simultaneously with a power-law, attenuated by two absorption components. The first accounts for the Galactic foreground absorption with a fixed column density of $6 \times 10^{20} \mathrm{~cm}^{-2}$ and solar abundances (Wilms et al. 2000), while the second models the absorption along the line of sight within the SMC and local to the source with free column density and reduced abundances of 0.2 for elements heavier than helium (Russell \& Dopita 1992). This resulted in an acceptable fit with reduced $\chi^{2}$ of 1.12 for 377 degrees of freedom (see Fig. 2). The best-fit value for the column density in the SMC was $1.8 \pm 0.2 \times 10^{21} \mathrm{~cm}^{-2}$ and the power-law photon index $0.74 \pm 0.02$, values typical of BeXRBs in the SMC (Haberl et al. 2008). The observed average flux $(0.2-10 \mathrm{keV})$ during the $X M M-N e w t o n$ observations was $1.4 \times 10^{-12} \mathrm{erg} \mathrm{cm}^{-2} \mathrm{~s}^{-1}$ (as obtained from the pn spectrum), which corresponds to a source luminosity corrected for absorption of $6.3 \times 10^{35} \mathrm{erg} \mathrm{s}^{-1}$ (assuming a distance of $60 \mathrm{kpc}$ ). The flux increased after the first observation and finally dropped by $27 \%$ in the last observation $\left(1.33 \times 10^{-12} \mathrm{erg} \mathrm{cm}^{-2} \mathrm{~s}^{-1}, 1.53 \times 10^{-12} \mathrm{erg} \mathrm{cm}^{-2} \mathrm{~s}^{-1}, 1.54\right.$ $\times 10^{-12} \mathrm{erg} \mathrm{cm}^{-2} \mathrm{~s}^{-1}$ and $1.13 \times 10^{-12} \mathrm{erg} \mathrm{cm}^{-2} \mathrm{~s}^{-1}$ in chronological order), which could indicate that we witnessed a typical type I outburst (Okazaki \& Negueruela 2001).

To investigate the soft extended emission, we extracted the EPIC spectra from a ring around the position of SXP 1062. The relatively wide wings in the point spread function of the $X M M-N e w t o n$ telescopes means a contribution from the X-ray pulsar to the soft emission cannot be avoided. Therefore, we modelled the spectrum of the diffuse emission with a powerlaw component in addition to a thermal component. We kept the SMC absorption and photon index for the power-law fixed at the values derived from the spectra of SXP 1062, allowing only a free normalisation. For the thermal component we used the plasma emission model mekal (also with SMC abundances as for the absorption) available in XSPEC (Mewe et al. 1985) with SMC absorption (free in the fit and different to that of the powerlaw component). The resulting best-fit parameters (reduced $\chi^{2}=$ 1.22 for 16 degrees of freedom) are $7.3_{-6.8}^{+8.8} \times 10^{20} \mathrm{~cm}^{-2}$ for the SMC absorption and $0.23 \pm 0.05 \mathrm{keV}$ for the temperature. The observed flux $(0.2-2 \mathrm{keV})$ of $1.1 \times 10^{-14} \mathrm{erg} \mathrm{cm}^{-2} \mathrm{~s}^{-1}$ makes

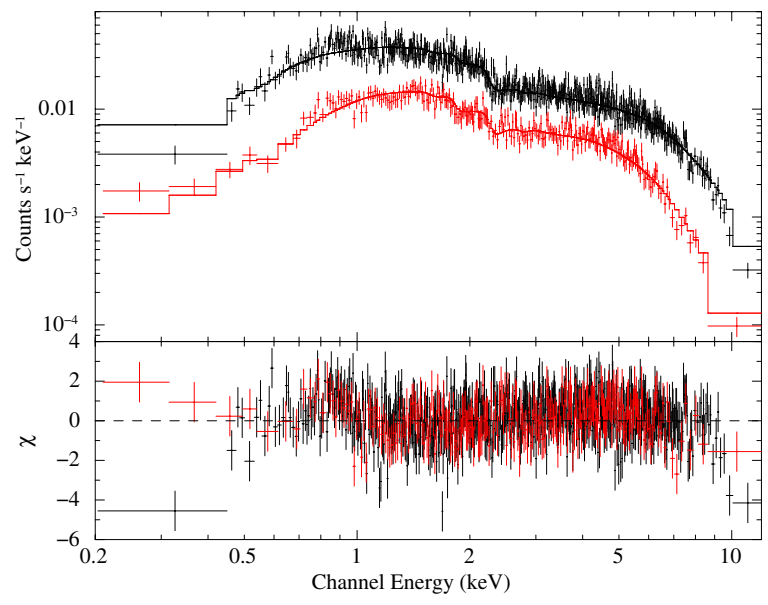

Fig. 2. EPIC-pn (black) and MOS2 (red) spectra of SXP 1062 combining the data from the four XMM-Newton observations. The best-fit ower-law model is plotted as histogram, and the lower panel shows the fit residuals.

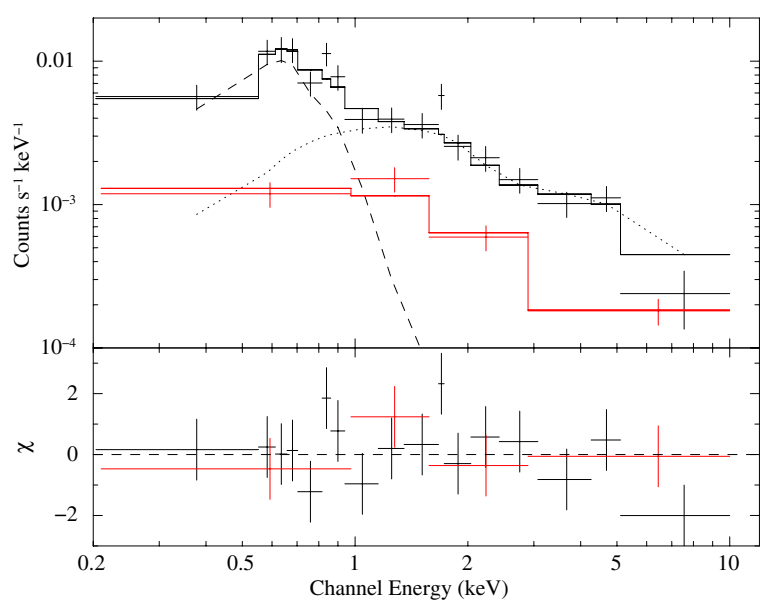

Fig. 3. EPIC spectra as in Fig. 2 extracted from the soft emission region around SXP 1062. The best-fit model is composed of a thermal (dashed line) and a power-law (dotted) component (see text).

it the X-ray faintest SNR known in the SMC (for other faint remnants see Filipović et al. 2008). The spectra with the bestfit model are presented in Fig. 3. Unfortunately, the low-quality statistics of the spectra do not allow a more detailed comparison of different models for the thermal spectral component.

The Hi column density of the SMC in the direction of SXP 1062 is measured to $2.1 \times 10^{21} \mathrm{~cm}^{-2}$ (Stanimirovic et al. 1999). The absorption inferred from the X-ray spectrum of SXP 1062 is similar, while for the soft emission region we can only place an upper limit of $\sim 1.6 \times 10^{21} \mathrm{~cm}^{-2}$. The fact that BeXRBs show source intrinsic absorption and the large uncertainty in the column density of the soft X-ray emission spectrum do not allow deriving any constraints on the radial position of the objects within the SMC. Nevertheless, the two column densities inferred from the X-ray spectra of the BeXRB and the diffuse $\mathrm{X}$-ray emission region (the SNR) are consistent with a location of the BeXRB within the SNR.

\subsubsection{Timing analysis of SXP 1062}

We performed a timing analysis of the EPIC light curves using the Bayesian approach (Gregory \& Loredo 1996) and a Rayleigh 


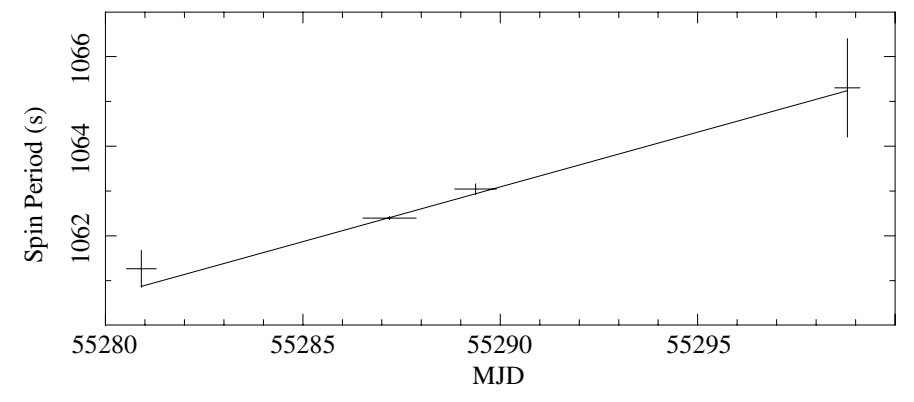

Fig. 4. Evolution of the spin period of SXP 1062 as obtained from the combined EPIC-pn and EPIC-MOS2 data of the four XMM-Newton observations. Spin periods and $1-\sigma$ errors were derived with the Bayesian odds ratio method.

$Z^{2}$ test for one harmonic (Haberl \& Zavlin 2002; Buccheri et al. 1983) around the known periodicity of 1062 s. To investigate a possible evolution of the spin period we first analysed each XMM-Newton observation separately. The obtained spin periods are plotted versus time in Fig. 4 and show a steady spin-down trend over the observing interval of about 18 days. A linear fit to the spin period evolution (line in Fig. 4) results in a period change $\dot{P}$ of $2.8_{-0.7}^{+0.8} \times 10^{-6} \mathrm{~s} \mathrm{~s}^{-1}\left(0.24_{-0.06}^{+0.07} \mathrm{~s} \mathrm{day}^{-1}\right)$. This value was used as starting point for a phase-coherent analysis combining the EPIC-pn data of all four observations searching a grid of different values for the spin period $P$ and a constant $\dot{P}$. We used the $Z^{2}$ test with one harmonic to account for the first harmonic seen in the FFT power density spectrum. This resulted in a maximum $Z_{1}^{2}$ value of 344.1 for $P=1061.24 \mathrm{~s}$ (at an epoch of MJD 55 280.53, the start of the first EPIC-pn exposure) and $\dot{P}=$ $3.0 \pm 0.5 \times 10^{-6} \mathrm{~s} \mathrm{~s}^{-1}$.

\section{Discussion and conclusions}

Our multi-wavelength morphological studies of the field around the new BeXRB pulsar SXP1062 confirm the previously unknown SNR proposed by HB11. Its SNR nature is further supported by its X-ray spectrum obtained from both the $X M M-N e w t o n$ EPIC data and its steep radio spectrum. The strong [O III] line emission suggests the SNR as oxygen-rich type (Arbutina \& Urošević 2005), which usually comes from a type Ib event, the explosion of a massive $\mathrm{O}, \mathrm{B}$, or WR star.

SXP 1062 is detected close to the centre of the SNR. At the outer edge of the eastern wing of the SMC, only very few BeXRBs are detected, and all known SNRs are located in the bar of the SMC (Payne et al. 2007; Filipović et al. 2008). This makes a chance coincidence of SXP 1062 with the SNR very unlikely. However, the ages of SNRs and BeXRBs are expected to be rather different. While SNRs can usually be seen for at most a few $10^{5}$ years in X-rays and radio (for the methods used to estimate SNR ages see e.g. Xu et al. 2005), BeXRBs are expected to become $\mathrm{X}$-ray active much later (see below). Using the relation $t_{\mathrm{y}}=3.8 \times 10^{2} R_{\mathrm{pc}}(k T)_{\mathrm{keV}}^{-1 / 2}$ from Xu et al. (2005) of temperature derived from X-ray spectral modelling $(0.23 \mathrm{keV})$ and the size of the SNR $\left(\sim 2.5^{\prime}\right.$ in diameter, corresponding to $\sim 40 \mathrm{pc}$ at a distance of $60 \mathrm{kpc}$ ), we estimate the age of the SNR to $\sim 16 \mathrm{ky}$. If one compares the ages estimated for SNRs in the SMC by van der Heyden et al. (2004) with their sizes as listed in Badenes et al. (2010), one finds ages of at most $25 \mathrm{ky}$ for remnants with diameters around 40-50 pc.

We estimate the distance between the BeXRB and the centre of the SNR to about $9^{\prime \prime}$, which corresponds to $\sim 8 \times 10^{13} \mathrm{~km}$ at SMC distance. From evolutionary investigations, kick velocities around 100-200 $\mathrm{km} \mathrm{s}^{-1}$ were invoked for neutron stars in HMXB systems (Portegies Zwart 1995). Using this as maximum space velocities for the binary systems and neglecting a velocity component in radial direction, it would take 13-26 ky for SXP 1062 to move from the SNR centre to its current position, compatible with the SNR age estimates from above, which are also consistent with those of HB11.

Statistical measurements of BeXRBs in the SMC result in lower space velocities: Coe (2005) inferred a lower limit of $30 \mathrm{~km} \mathrm{~s}^{-1}$ from measuring the separation of BeXRBs to nearby young stellar clusters. Similarly Antoniou et al. (2010) argue from the correlation between the number of BeXRBs and the local star formation rate $42 \mathrm{My}$ ago at their current position for a maximum velocity of $15-20 \mathrm{~km} \mathrm{~s}^{-1}$. Both cases should actually be regarded as minimum velocities as the BeXRB might not have been born in the nearest star cluster. The work of Knigge et al. (2011) might also suggest that BeXRB pulsars with long spin period received stronger kicks. In either case, using lower space velocities (and also components in radial direction) would correspondingly increase the SNR age estimate.

According to the model calculations of Urpin et al. (1998), it seems very unlikely that an NS can be spun down to a period around $1000 \mathrm{~s}$ within a few $10^{4}$ years if it was born with a spin period around $10 \mathrm{~ms}$. Of course, such model calculations assume simplified approximations of the conditions in a BeXRB (in particular concerning the highly variable accretion usually observed in such systems), but only for models with extreme conditions (high magnetic field strength for the NS and low accretion rates), spin periods in excess of a few $100 \mathrm{~s}$ are reached after more than $10^{6}$ years. If the NS in SXP 1062 is indeed the compact remnant from the supernova explosion, which also created the SNR around it, then either the NS was born with a much longer spin period than $10 \mathrm{~ms}$, or some other effect plays an important role to efficiently decelerate the rotation of the NS.

SXP 1062 was discovered as a transient BeXRB pulsar during XMM-Newton and Chandra observations in 2010 when its $\mathrm{X}$-ray luminosity was at the level of $6 \times 10^{35} \mathrm{erg} \mathrm{s}^{-1}$. The BeXRB was not active during the ROSAT PSPC observations of the SMC (Haberl et al. 2000). Based on the longest PSPC observation from 1991-10-07 and assuming the spectral shape measured by EPIC, we derive an upper limit of 1.5 $\times 10^{-13} \mathrm{erg} \mathrm{cm}^{-2} \mathrm{~s}^{-1}(0.2-10 \mathrm{keV})$, assuming an upper limit of $10 \mathrm{cts}$ and a vignetting-corrected exposure of $9.7 \mathrm{ks}$ at the position of SXP 1062. This is about a factor of 10 lower than the flux during the XMM-Newton observations. While BeXRB transients generally exhibit spin-up during intervals of increased accretion (e.g. Bildsten et al. 1997), SXP 1062 is peculiar as it showed a strong spin-down consistent with a constant rate of $0.26 \mathrm{~s} \mathrm{day}^{-1}$ over 18 days. Coe et al. (2010) investigated RXTE monitoring data covering $\sim 10$ years to study the spin period changes of BeXRB pulsars in the SMC over their active periods, which typically last 50-500 days. They find four out of 15 systems with measured short-term spin period changes, which showed spindown during outburst. In Table 1, we summarise the pulse period changes for some X-ray binary pulsars, which show times of spin-down. We list the frequency derivative $\dot{f}=-\dot{P} / P^{2}$, which is directly related to the accretion torque exerted on the NS. We include the three SMC BeXRB pulsars with well measured values of $\dot{P}$ and for comparison Cen X-3 (a super-giant HMXB with Roche-lobe overflow) and GX 1+4, a low-mass system with M5 III donor star. SXP 1062 exhibits spin-down about five times larger than the other listed SMC BeXRBs (which have remarkably similar values). It should be noted that the values derived by Coe et al. (2010) are average values over longer periods of time 
Table 1. X-ray binary pulsars with periods of spin-down.

\begin{tabular}{lccc}
\hline \hline $\begin{array}{c}\text { Object } \\
\text { name }\end{array}$ & $\begin{array}{c}\text { Pulse } \\
\text { period }[\mathrm{s}]\end{array}$ & $\begin{array}{c}\dot{f} \\
{\left[\mathrm{~Hz} \mathrm{~s}^{-1}\right]}\end{array}$ & Reference \\
\hline Cen X-3 & 4.82 & $-(1-5) \times 10^{-12}$ & Bildsten et al. (1997) \\
SXP 8.80 & 8.90 & $-5.1 \times 10^{-13}$ & Coe et al. (2010) \\
GX 1+4 & 110.2 & $-2.1 \times 10^{-13}$ & Makishima et al. (1988) \\
SXP 144 & 144.5 & $-5.9 \times 10^{-13}$ & Coe et al. (2010) \\
SXP 1062 & 1062 & $-2.6 \times 10^{-12}$ & this work \\
SXP 1323 & 1325 & $-5.0 \times 10^{-13}$ & Coe et al. (2010) \\
\hline
\end{tabular}

and that intermittent intervals of spin-up (as usually seen from accreting pulsars, Bildsten et al. 1997) reduce the longer-term average.

SXP 1062 shows a remarkably large accretion torque, similar in magnitude to that of Cen X-3. In this HMXB intervals of steady spin-up and spin-down alternate, which last typically 10-100 days (Bildsten et al. 1997), but at much higher X-ray luminosities of $\sim 10^{38} \mathrm{erg} \mathrm{s}^{-1}$ (e.g. Nagase et al. 1992). Also GX 1+4 was still observed at $10^{36} \mathrm{erg} \mathrm{s}^{-1}$ during its extended lowstate (Makishima et al. 1988), about a factor of 2 brighter than SXP 1062. This demonstrates that the generally accepted model for accretion, where the torque is directly proportional to the mass accretion rate (hence luminosity) is probably too simple, as similar torques can be exerted, although the luminosity is more than a factor of 100 different. The work of Bildsten et al. (1997) suggests that disk-accreting pulsars are subject to instantaneous torques with similar magnitude but opposite sign. Different average long-term spin-up or spin-down values of individual pulsars would then be the result of different time scales for reversals between spin-up and -down.

SXP 1062 most likely also shows intervals of alternating spin-up and spin-down, which would reduce the magnitude of the long-term spin change. Assuming the extreme case that the NS was spun down with $-2.6 \times 10^{-12} \mathrm{~Hz} \mathrm{~s}^{-1}$ $\left(-8.4 \times 10^{-5} \mathrm{~Hz} \mathrm{y}^{-1}\right)$ over its whole life of $25 \mathrm{ky}$ (the maximum age) an upper limit of $2 \mathrm{~Hz}$ for the total spin-down is derived, which corresponds to a lower limit of $0.5 \mathrm{~s}$ for the spin period at birth. For a more realistic, smaller long-term average accretion torque a correspondingly longer birth spin period is expected. Therefore, if the NS in SXP 1062 is indeed the compact remnant from the supernova explosion that created the SNR, our results show that the NS was most likely born with a spin period much longer than a few tens of ms as generally adopted for an NS at birth.

We confirm the existence of a new SNR around the BeXRB SXP 1062 in the SMC, independently suggested by Henault-Brunet et al. (2011). The SNR is detected in optical, radio, and X-ray wavelengths and shows a shell-like structure with the BeXRB close to its projected centre. We estimate an age of around 10-25 ky for the remnant. Such a time scale is too short to spin down the neutron star in a BeXRB to $\sim 1000 \mathrm{~s}$ if it was born with a canonical spin period of a few $10 \mathrm{~ms}$. SXP 1062 is remarkable for showing a strong average spin-down rate $\dot{f}$ of $-2.6 \times 10^{-12} \mathrm{~Hz} \mathrm{~s}^{-1}$ during the XMM-Newton observations distributed over 18 days, while it was detected during high X-ray activity at $6.3 \times 10^{35} \mathrm{erg} \mathrm{s}^{-1}$. Assuming the extreme case that such a high reverse accretion torque was exerted on the neutron star for its whole life, a lower limit of $0.5 \mathrm{~s}$ for the birth spin period is inferred. If the neutron star in SXP 1062 is indeed the compact remnant from the supernova explosion that created the SNR, we conclude that the neutron star in SXP 1062 was born with an even longer spin period for more realistic, lower longterm average accretion torques.

Acknowledgements. The XMM-Newton project is supported by the Bundesministerium für Wirtschaft und Technologie/Deutsches Zentrum für Luft- und Raumfahrt (BMWI/DLR, FKZ 50 OX 0001) and the Max-Planck Society. The MCELS data are provided by R. C. Smith, P. F. Winkler, and S. D. Points. The MCELS project has been supported in part by NSF grants AST-9540747 and AST-0307613, and through the generous support of the Dean B. McLaughlin Fund at the University of Michigan, a bequest from the family of Dr. Dean B. McLaughlin in memory of his lasting impact on Astronomy. The National Optical Astronomy Observatory is operated by the Association of Universities for Research in Astronomy Inc. (AURA), under a cooperative agreement with the National Science Foundation. We used the KARMA and MIRIAD software package developed by the ATNF. The ATCA is part of the Australia Telescope, which is funded by the Commonwealth of Australia for operation as a National Facility managed by CSIRO. R.S. acknowledges support from the BMWI/DLR grant FKZ 50 OR 0907

\section{References}

Antoniou, V., Zezas, A., Hatzidimitriou, D., \& Kalogera, V. 2010, ApJ, 716, L140

Arbutina, B., \& Urošević, D. 2005, MNRAS, 360, 76

Badenes, C., Maoz, D., \& Draine, B. T. 2010, MNRAS, 407, 1301

Bhattacharya, D., \& van den Heuvel, E. P. J. 1991, Phys. Rep., 203, 1

Bildsten, L., Chakrabarty, D., Chiu, J., et al. 1997, ApJS, 113, 367

Buccheri, R., Bennett, K., Bignami, G. F., et al. 1983, A\&A, 128, 245

Coe, M. J. 2005, MNRAS, 358, 1379

Coe, M. J., Edge, W. R. T., Galache, J. L., \& McBride, V. A. 2005, MNRAS, 356,502

Coe, M. J., McBride, V. A., \& Corbet, R. H. D. 2010, MNRAS, 401, 252

Corbet, R., Markwardt, C. B., Marshall, F. E., et al. 2003, IAU Circ., 8064, 4

Filipovic, M. D., Haynes, R. F., White, G. L., et al. 1995, A\&AS, 111, 311

Filipovic, M. D., Haynes, R. F., White, G. L., \& Jones, P. A. 1998, A\&AS, 130, 421

Filipović, M. D., Payne, J. L., Reid, W., et al. 2005, MNRAS, 364, 217

Filipović, M. D., Haberl, F., Winkler, P. F., et al. 2008, A\&A, 485, 63

Ghosh, P., \& Lamb, F. K. 1978, ApJ, 223, L83

Gregory, P. C., \& Loredo, T. J. 1996, ApJ, 473, 1059

Haberl, F., \& Pietsch, W. 2005, A\&A, 438, 211

Haberl, F., \& Zavlin, V. E. 2002, A\&A, 391, 571

Haberl, F., Filipović, M. D., Pietsch, W., \& Kahabka, P. 2000, A\&AS, 142, 41

Haberl, F., Eger, P., \& Pietsch, W. 2008, A\&A, 489, 327

Henault-Brunet, V., Oskinova, L. M., Guerrero, M. A., et al. 2011, MNRAS in press [arXiv: 1110.6404]

Hughes, J. P., \& Smith, R. C. 1994, AJ, 107, 1363

Israel, G. L., Campana, S., Covino, S., et al. 2000, ApJ, 531, L131

Knigge, C., Coe, M., \& Podsiadlowski, P. 2011, Nature, 479, 372

Makishima, K., Ohashi, T., Sakao, T., et al. 1988, Nature, 333, 746

Mewe, R., Gronenschild, E. H. B. M., \& van den Oord, G. H. J. 1985, A\&AS, 62, 197

Nagase, F., Corbet, R. H. D., Day, C. S. R., et al. 1992, ApJ, 396, 147

Okazaki, A. T., \& Negueruela, I. 2001, A\&A, 377, 161

Owen, R. A., Filipović, M. D., Ballet, J., et al. 2011, A\&A, 530, A132

Payne, J. L., White, G. L., Filipović, M. D., \& Pannuti, T. G. 2007, MNRAS, 376,1793

Portegies Zwart, S. F. 1995, A\&A, 296, 691

Reig, P. 2011, Ap\&SS, 332, 1

Russell, S. C., \& Dopita, M. A. 1992, ApJ, 384, 508

Shtykovskiy, P., \& Gilfanov, M. 2005, MNRAS, 362, 879

Stanimirovic, S., Staveley-Smith, L., Dickey, J. M., Sault, R. J., \& Snowden, S. L. 1999, MNRAS, 302, 417

Strüder, L., Briel, U., Dennerl, K., et al. 2001, A\&A, 365, L18

Turner, M. J. L., Abbey, A., Arnaud, M., et al. 2001, A\&A, 365, L27

Urpin, V., Konenkov, D., \& Geppert, U. 1998, MNRAS, 299, 73

van der Heyden, K. J., Bleeker, J. A. M., \& Kaastra, J. S. 2004, A\&A, 421, 1031

Wilms, J., Allen, A., \& McCray, R. 2000, ApJ, 542, 914

Wong, G. F., Filipovic, M. D., Crawford, E. J., et al. 2011, Serbian Astron. J., 182,43

Xu, J.-W., Zhang, X.-Z., \& Han, J.-L. 2005, Chinese J. Astron. Astrophys., 5, 165

Ye, T., \& Turtle, A. J. 1993, in Lecture Notes in Physics (Berlin: Springer Verlag), New Aspects of Magellanic Cloud Research, ed. B. Baschek, G. Klare, \& J. Lequeux, 416, 167 